\title{
Stigma as a Barrier to HIV Prevention among an Indian-Immigrant Population in Canada: A Qualitative Study
}

\section{Basanti Majumdar*}

Faculty of Health Sciences, McMaster University, Canada

\begin{abstract}
Purpose: To broaden understanding of the vulnerability to HIV of Indian immigrant communities in Canada. This study focused on the exploration of (a) selected population of Indian immigrants' perceptions of HIV risk, risk behaviour, and utilization of HIV/AIDS-related services, and (b) the effects of cultural norms of these communities in shaping their capacity for and experiences of responding to HIV in Canada.
\end{abstract}

Methods: A total of 24 semi-structured interviews were conducted with selected population of the Indian-immigrant community. Participants were recruited purposively through a South Asian Punjabi community health service. Each interview lasted from one to two hours and was conducted in English or Punjabi depending on the preference of the participant. Tapes were transcribed verbatim and translated to English. Interview transcripts were coded and analyzed using N-Vivo software. Final themes and sub-themes were decided on by consensus among researchers.

Results: Participants discussed cultural taboos and stigma associated with any topic that was perceived to be connected to sexual behaviour. Participants identified that these cultural taboos and the resulting stigma has reduced opportunities for members of the Indian immigrant population to obtain accurate information related to HIV. Although participants agreed that knowledge of HIV was important for prevention, participants discussed a reluctance to talk openly about issues or to seek information publically.

Conclusions: Social taboos that limit discourse around sexuality and sexual behaviour negatively impact the effectiveness of HIV prevention education efforts which have targeted mainstream Canadians. This study points to the need for innovative strategies that address social norms and influences among a diverse group of people who maintain close ties with family and friends living in India. Social network theory can provide guidance for carefully planned peer outreach initiatives which may provide an effective means to reach this underserved community.

Keywords: Behaviour; Knowledge; Risk; Beliefs; Social network theory

\section{Background}

In 2008, an estimated 2.27 million people in India between the ages of 15-49 years were living with HIV. Although the HIV prevalence in India is below $1.0 \%$, this country carries the largest burden of HIV behind South Africa and Nigeria [1,2]. The HIV epidemic in India is now entering a phase where HIV transmission is increasingly occurring within intimate partner sexual relationships. Unprotected heterosexual transmission accounts for the largest proportion of HIV cases (87\%). Women account for a growing proportion of the total HIV infected population and more than $90 \%$ of these women acquired HIV infection from their husbands or intimate sexual partners [1].

In 2006, South Asians became Canada's largest visible minority group, representing one-quarter (24.9\%) of all visible minorities [3]. The term South Asian refers to a diverse group of people originating from countries across the Indian subcontinent, including India, Pakistan, Nepal, Sri Lanka, and Bangladesh (Statistics Canada, 2006). India is the home-country of the largest proportion of South Asian immigrants arriving to Canada [4]. In 2009-2010, over 28,000 new Indian immigrants arrived in Canada [5].

In Canada, the number of people living with HIV (including AIDS) continues to rise, from an estimated 64,000 in 200 to 71,300 in 2011 (an 11.4\% increase) [6]. HIV case report data with race/ethnicity information has been collected since 1998. National trends must be interpreted with caution, however, as not all provinces (Ontario included) report positive HIV tests with race/ethnicity information. In Canada, $42.4 \%$ of positive HIV test reports with race/ethnicity information were attributed to the White racial/ethnic category, and $32.7 \%$ to an Aboriginal category. This was followed by the Black racial/ ethnic category (at 11.8\%); Asian (6.8\%), Latin American (2.9\%), and South Asian/West Asian/Arab (2.2\%) [7]. While the proportion of South Asian population living with HIV/AIDS in Canada is small, it is important to note that the number of cases nationally continues to increase. Improved reporting mechanisms and surveillance is required to supply more accurate data.

The 2002 Ontario Advisory Committee on HIV/AIDS strategic plan emphasizes the need to determine the impact of factors such as attitudes, values, and marginalization of ethnic groups, which may contribute to increased risk of poor health. Education regarding HIV/ AIDS is lacking in South Asian-immigrant communities, and access to accurate information and culturally competent treatment is limited [8] Particularly, women from South Asian-immigrant communities may continue to be at an increased risk for contracting HIV due to their inability to read and their lack of access to accurate information $[9,10]$.

Despite a growing South Asian-immigrant community in Canada, little research has examined the relationship between this population of newcomer and second-and third-generation migrants, and HIV risk. Although South Asian-immigrant communities in Canada are

*Corresponding author: Basanti Majumdar, McMaster University, Faculty of Health Sciences Room No.3N28, 1280 Main Street West, Hamilton, Ontario, Canada L8S 4K1, Baldev Mutta, Executive Director, Punjabi Community Health Services, 11730 Airport Road, Brampton, ON, L6R 0C7, Canada; E-mail: Majumdar@mcmaster.ca

Received May 28, 2013; Accepted July 12, 2013; Published July 15, 2013

Citation: Majumdar B (2013) Stigma as a Barrier to HIV Prevention among an Indian-Immigrant Population in Canada: A Qualitative Study. Primary Health Care 3: 137. doi:10.4172/2167-1079.1000137

Copyright: () 2013 Majumdar B. This is an open-access article distributed under the terms of the Creative Commons Attribution License, which permits unrestricted use, distribution, and reproduction in any medium, provided the original author and source are credited. 
linguistically, culturally, and religiously diverse, the majority of them living in Canada report maintaining contact with overseas family members through visits, letters and telecommunications, and for decades after arrival in Canada. Furthermore, over $80 \%$ of South Asians in Canada report that maintaining ethnic customs and traditions is important, or very important, to them [4]. It is widely accepted that culture and social customs determine norms surrounding sexual discourse and sexual behaviour [11]. South Asian immigrants come to Canada with values related to sexuality, such as not discussing the word "sex" openly, and not sharing the knowledge of sexuality and its relationship with the causes of HIV/AIDS. Many of their beliefs related to sexual practice come from their experience and knowledge from their lives in India.

According to social network theory, individuals function within social networks that establish, and enforce, norms for behaviour [12]. This theory takes into account that individuals do not make decisions in isolation but interact with others from their social network [13]. Social norms function as points of reference for social groups, and are a source of social comparison, control, and influence [14]. Social networks may establish and enforce norms relevant to HIV-risk by creating norms that are supportive of some behaviours while stigmatizing others. Dependent on the norms that are created, these norms can either slow or facilitate the spread of HIV $[12,15,16]$. In this study, we explore social norms that inhibit discourses about knowledge and awareness of HIV/AIDS.

Currently, there is a lack of research into the impact of the cultural norms related to sexual discourse on the ability of South Asian -immigrant communities to prevent the spread of HIV/AIDS. A small body of literature has identified a general lack of openness surrounding sexual discourse among South Asian cultures which may interfere with the development of skills to negotiate safe sex practices $[8,17-19]$. The current study expands the existing body of knowledge and provides insight into to how social norms limiting sexual discourse among the South Asian -immigrant community in Canada act as a barrier to the prevention of HIV/AIDS.

\section{Methods}

\section{Purpose}

The purpose of the current study was to increase understanding of the vulnerability to HIV among Indian immigrant communities in Canada. This study focused on the exploration of (a) selected community of Indian immigrants' perceptions of HIV risk, risk behaviour, and utilization of HIV/AIDS-related services, and (b) the effects of cultural norms of these communities in shaping their capacity for and experiences of responding to HIV in Canada.

\section{Ethics}

Ethics approval for this study was granted from McMaster University's Research Ethics Board as well as the boards of the collaborating South Asian community organization.

\section{Sample}

A total of 24 key informants from the Greater Toronto area and City of Hamilton were interviewed for this study, 13 males and 11 females. Key informants were defined as individuals who are themselves members of the Indian-immigrant community and who are knowledgeable about their community. Participants were recruited purposively through multiple networks of Indian immigrants (e.g.: self-organized immigrant groups, relevant websites, newspapers, HIV/
AIDS-related social and health services, as well as non-governmental organizations). All participants reported having immigrated to Canada from the Punjab region in Northern India.

\section{Interviews}

Semi-structured in-depth individual interviews were conducted face-to-face with all participants. An interview guide was developed by the research team which consisted of open-ended questions designed to elicit rich descriptions. Questions were developed to explore themes of interest, including HIV knowledge, perceptions about HIV risks, relationships with (same-sex or heterosexual) partners, decisionmaking on safe-sex, other risk-taking behaviours (e.g.: injection drug use), knowledge about HIV-related services available, and help-seeking behaviour. The interview guide was tested on a sample of 8 men and 8 women from the Indian-immigrant community and modified based on feedback. The interview guide was then translated into Punjabi and back-translated to English to ensure validity.

Potential participants were invited to participate in the study. Willing participants were given an introductory telephone interview which assisted in developing rapport between the interviewer and participant. During the introductory telephone interview, a time and date were fixed for the face-to-face in-depth interview. Before the indepth interview began, signed informed consent was obtained from the participant. Most of the interviews were conducted in the Punjabi health service centre where a private room was booked for the interview session. Some of the interviews were conducted in a coffee shop or restaurant nearer to the interviewee's residence, and as preferred by the participant. Each interview lasted from one to two hours and was conducted by a hired interviewer who could communicate in English, as well as three languages common to Northern India: Hindi, Urdu, and Punjabi. The interviewer was trained by the research team and interviews were conducted in English or Punjabi depending on the preference of the participant. Participants were provided with an honorarium for participating in the study.

\section{Timeline}

Participant recruitment and interviews were conducted during a six-month period spanning 2008 and 2009.

\section{Data analysis}

All interviews were tape-recorded with the permission of the participants. Tapes were transcribed verbatim, and those conducted in Punjabi were translated to English. A translator was hired to carry out translation of the transcripts. Based on Leininger's [20] method for reliability and validity of the translation of interview guides, the questions were translated from English to Punjabi by a community expert selected by the Punjabi health services. A second person was also selected by the same agency to translate back the questions to English. A third person was selected to examine if the questions carry the same meaning and wordings, and are acceptable to the Punjabi community. All three of these persons are comfortable in both languages. The translated documents were validated by the interviewer to make sure that statements and expressions were translated appropriately. The interview data was entered into N-Vivo software, and the research team analysed transcripts with grounded theory [21]. Transcripts were coded, with attention to emerging concepts. Constant comparisons were drawn to arrive at higher order categories, or themes. The final themes and sub-themes were determined by consensus. 


\section{Results}

The major finding of this study is that due to the close relationship between sexual behaviours and HIV transmission, stigma and cultural taboos presented a fundamental barrier to the prevention of HIV through lack of knowledge and misperceptions, and limited helpseeking behaviour.

\section{Stigma}

Throughout the interviews, participants discussed stigma associated with discourse around sexual behaviour. As the majority of participants characterize HIV as a disease that is transmitted sexually, cultural taboos and stigma extended to HIV.

“...I think the first response you would get is that you get [HIV] out of sex. You are doing something, you shouldn't be doing. That's probably why it is looked at as taboo, you don't talk about it, and you don't hear about it." (Participant 123)

Some participants expressed difficulty discussing the topic of sexual behaviour and HIV themselves during the interview:

"I don't know much. I know they say you get it from having sex (giggles). I feel shy talking about it (laughs). You're the age of my child, like my child. I feel shameful talking with you about [sex]." (Participant 107)

The stigma around HIV and its relationship to sexual behaviour has risen from cultural taboos around sex, especially for women. Participants identified that cultural taboos and stigma have reduced opportunities for members of the Indian immigrant population to obtain accurate information related to HIV and HIV prevention:

"...many barriers. The women that have come from India especially don't go outside for sex knowledge. What if the community finds out what would they say? Nobody talks about this openly." (Participant 113)

“...culture has played a big role; everyone feels embarrassed and ashamed talking about sex.... Even the female professors wouldn't talk about it they felt shy and embarrassed." (Participant 118)

\section{Lack of Knowledge and Misunderstandings about HIV}

Participants varied in the amount of correct knowledge they had about HIV and HIV prevention. The majority of participants expressed that they had "very little" knowledge about the disease. While all of the participants knew that HIV could be transmitted through sexual behaviour, fewer knew about other modes of transmission, including blood transfusions, sharing needles, and mother-child. Participants also described the knowledge of their families and community members as limited:

"Like general knowledge yes they know that this disease exists that harms your body, can ruin your entire immune system but not more knowledge than this. Like how to contract it, what its cures are, what the medications are - they don't know this." (Participant 108)

"Generally they only think if you have sex in the wrong ways you can get it. They have a general idea but they don't have any awareness nor does anyone speak about it openly."(Participant 100)

In addition to a lack of knowledge, participants also revealed misperceptions about the transmission of HIV among the Indian immigrant community. Participants discussed a lack of understanding related to HIV among both the immigrant community and their social network in India. Misperceptions that were identified by participants included beliefs that (1) community members could not catch HIV, (2) community members do not engage in risky sexual behaviours, and (3) transmission occurs through casual contact:

"Our community doesn't believe that we can get it too." (Participant 105).

“They think our children don't do things like this, so why would they want to learn about it. It's only when they start realising that there is a big outbreak in India, which I found out. They still say here that those people are low-class so it won't affect us." (Participant 120)

"...they think its spread like the flu by breathing or contact. When they find out a person has it, they don't understand it." (Participant 124)

Participants attributed this lack of awareness and misunderstanding to broader cultural norms and the stigma attached to discourse around sexual behaviour, sexual behaviour, and related topics:

"It's not a subject to talk about. It's not a subject they are comfortable with. Honestly I haven't heard anybody around me or within the family or anybody else really talking about it... You kind of hear about it in the news. There is far more ignorance about it and how it is caused and stuff." (Participant 123)

"I think our culture they give you a girl to marry whose good and that's it they think you will have no problem at all with HIV/AIDS. Because in our culture we're not people that go out and have multiple partners. We believe that you get married once, that's your partner and that's it. So you don't really get exposed to it, and she thinks she's not getting exposed to it because she believes her husband is only with her. If he goes outside the marriage that's the only way he'll get AIDS." (Participant 120)

“... also realize there are taboos about certain things and they won't talk about unless it really affects them. They will try not to speak about it or they'll have little knowledge of it because no one close to them has that infection or disease, so they tend to - its taboo you know. If we're not actually affected by it then why talk about it. If you're talking about it with elders they'll just tell you to be quiet and that it is nonsense and ignore it and bypass the subject kind of thing. They're not actually going to sit down and say let's talk about it or even talk about normal sex. They don't want to talk about teenagers having sex... They think it's somebody else's duty; it's not our duty to discuss it. And then when a teacher talks about we're teaching sexual education at school, they'll turn around and say why they are talking about it even in school. Obviously they know why they have to talk about it, but there's such a taboo put on it from our cultural side they won't openly admit that they do talk about it or discuss it." (Participant 116)

Throughout the interviews a lack of knowledge regarding HIV was apparent. Participants self-identified as having little knowledge about HIV and expressed that even less correct knowledge was held among family and community members. Apparent in this discussion was the relationship between lack of knowledge, cultural norms, stigma regarding sexual behaviour and the resultant lack of discussion about this topic. Due to the lack of knowledge and stigma associated with this topic, participants discussed limited information-seeking behaviours among the community members.

\section{Limited Information-Seeking Behaviours}

There was a general consensus among study participants that obtaining accurate information related to HIV was important. Accurate information was seen as key to the protection of themselves, their family, and their community. However, the majority of participants 
stated that were unaware of information available to them and that they had not accessed HIV-related services.

"I just feel our community lacks knowledge and there's nowhere to get it, where you feel satisfied that you know enough to feel safe about it." (Participant 102)

Some participants expressed a reluctance to obtain information for fear of what people would think:

Interviewer: "Have you ever sought our accessed HIV information in Canada?"

Participant 105: "No I felt ashamed because people would think that I have it."

Interviewer: "Do you think this is important information to provide to the public?"

Participant 105: "Yes I do because my community doesn't know about it and it's important."

Participants discussed the interconnection between cultural norms, stigma and misperceptions and their impact on community member's knowledge seeking behaviours:

"Well for one [Indian women] just don't know where to get the information from. They don't go anywhere and so they don't open up networks for information. They don't read they just focus on the homely duties. The ones who get information go out to you know, like they have some interest to get this information. Being able to talk to their kids about it, or just the fact that they should know. Some ladies just don't have access because they might not drive, or they don't know how to speak English, so they go everywhere with their husbands." (Participant 101)

“There isn't anywhere to get information because a family member comes with you. You would have to explain exactly where you're going and they wouldn't approve of you getting that kind of information." (Participant 105)

"But now if you look at the way society has changed if a girl doesn't have a boyfriend it's a bad thing. These kids have no knowledge about sex; they keep many partners, and have no knowledge. I think India has more of a problem with HIV than they do here. If you have a partner, you are definitely going to have sex, but nothing is explained. And everything is kept secret from your parents whether you're from the village, or the city." (Participant 112)

Of key concern for many participants was the protection of their children from HIV. Participants discussed a desire for more knowledge related to HIV so that they could better inform their children. However, participants struggled with the desire to teach their children about how to protect themselves from HIV on the one hand and the cultural norms limiting discourse around sexual behaviour on the other:

"Parents don't talk about it. They think you shouldn't speak to your children about sex, and it should be hidden. And if the kids don't know about it, how will they be safe? The community doesn't discuss it, the parents don't discuss it, so who are you suppose to talk to it about? When you go to the doctor your mother is there with you $90 \%$ of the time. A 13 year old girl doesn't go to see her doctor without her mother, so she can't even ask her doctor. Otherwise her mom will find out she has sex and she'll be in trouble since our culture doesn't allow it, our culture says that there should be no sex before marriage. Then the risk increases. Everyone thinks their child isn't doing it and the information isn't passed on that they need to be safe." (Participant 101)
Interviewer: "Growing up did your family ever discuss this issue?"

Participant 124: "No never."

Interviewer: "Do you talk to your kids about it?"

Participant 124: "No, because this illness is sexual."

Acculturation to Canadian society may play a role in the ability of parents to address HIV prevention with their children. Participants whose families had been in Canada longer expressed confidence in their ability to address sexual behaviour and HIV prevention with their children:

"We have that being second or third generation born outside we have that openness, you know we can talk about certain things... But if the kids are talking about sex, it's not going to be a big thing, I'm not going to say go ask your mom kind of thing, I'll talk to them frankly about it." (Participant 116)

Stigma and cultural norms related to discourse around sexual behaviour combined with misunderstanding about HIV work together to create a climate that results in limited information seeking behaviour. Limited information seeking behaviour reinforces lack of knowledge and misunderstandings around HIV. The result is two-fold: (1) limited understanding and use of protective measures to prevent HIV transmission, and (2) limited knowledge dissemination from parents to children.

\section{Discussion}

Cultural taboos and stigma surrounding open discourse about sexual practices and sexual behaviour have resulted in misunderstandings about HIV transmission as well as a lack of information-seeking behaviour among the selected Indian-immigrant community in Canada. Social taboos that limit discourse around sexuality and sexual behaviour negatively impact the effectiveness of HIV prevention education efforts which are targeted toward mainstream Canadians. The data above reveals the gendered nature of cultural norms that may prevent South Asian women and men from openly acknowledging and discussing sexuality and sexual diseases with their peers and children. Abrahams [17] discusses the social norms that characterize sexuality and sexual behaviour in India. She notes that cultural norms are especially rigid for young women, who are assumed to be virgins when married, and where monogamy is assumed. Boys, on the other hand, are beginning to have greater access to information about sex, and more sexual experiences in their youth. While sexual discourse and behaviours are becoming less conservative in India as elsewhere, the result is increasing tensions between the genders and the generations as traditional notions of social norms come into conflict with more modern norms. Similarly in Canada, social norms about sexuality are changing from generation to generation, and the change is even more abrupt for families who migrate to Canada to find themselves subject to a dominant discourse about sexuality and sexual behaviour that is radically different from the traditions of home.

The participants interviewed here reveal that traditional social norms still guide the discourse around sexuality and knowledge about HIV/AIDS, and that these norms are highly gendered. The participants acknowledge the extent to which stigma and shame are associated with speaking openly about sexuality and HIV/AIDS. The ability to speak about these topics are limited for most men, but even more so for women. Interestingly, Abrahams [17] noted that the young girls in Mumbai had quantifiably fewer words (terminology and slang) to describe sexuality and sexual experiences compared to the boys. This 
suggests that women and girls are subject to a higher degree of control around access to information and communication about sexual health, due to the social norms that govern their understanding, awareness and knowledge about their bodies and sexuality. Gendered and generational differences influence the ability to communicate with others about sexuality and HIV/AIDS, so that the participants have differential knowledge, awareness, and access to information about HIV/AIDS prevention. Second and third generation South Asian youth may be adopting the sexual norms of their Canadian-born peers, while their parents are still governed by traditional social norms that leaves them silent on the topic of sexuality. Without some way of accessing reliable health information, the women and teens in these families may be at greater risk for HIV/AIDs.

HIV-prevention may be particularly difficult if even discussion about HIV and sexual behaviour is considered unacceptable [14]. To be effective, HIV-prevention interventions need to be powerful enough to counteract prevailing social norms. In the context of strong social norms that limit discussion of sexuality and sexual behaviour, traditional approaches to HIV-prevention which focus on education and skills building so that individuals can make rational health behaviour decisions, may not be effective. Bhattacharya [9] argues for health promotion approaches that privilege community-based rather than individualistic strategies for South Asian communities. A social network approach to the prevention of HIV has more promise as this approach aims to change social norms, including the acceptability of talking about HIV and sex, by focusing on access to risk-reduction materials and targeting persons who have social influence [14]. Under a social network approach to HIV prevention, peer outreach initiatives may serve as a powerful tool for challenging and changing social [15]. According to social network theory, social network members who are widely respected and have the power to establish and enforce social norms, have a critical role to play in changing social norms that act as a barrier to HIV prevention. A social network approach to HIV prevention seeks to change social norms that act as barriers to HIV prevention and capitalize on existing social norms that are favourable to preventive behaviour by enlisting the aid of natural opinion leaders [12]. To develop the outreach initiative, it is necessary to identify, through social network analysis, the network members who have the most influence on adults and youth, and to determine the most effective ways to enhance communication about HIV/AIDS among the members of this social group. The community health agencies would be a logical starting place to enlist opinion leaders. As identified in this research, traditional social norms are in conflict with more modern norms, which may necessitate an approach to social norm change that targets separate age groups (youth and adults). Any efforts to improve awareness about and prevention of HIV/AIDS in South Asian communities in Canada would also be strengthened by an analysis of youth and adult sexual behaviour so that promotional strategies could be targeted effectively.

\section{Conclusion}

It is difficult to make any generalization based on this study, as the population is a selective group with background of Punjabi community of northern India. India has a much diversified heterogeneous culture based on the provincial and religious background. Based on this study it can be said that social taboos that limit discourse around sexuality and sexual behaviour negatively impact the effectiveness of HIV prevention education efforts which have targeted mainstream Canadians. This study provides insight into the lack of HIV-related knowledge and information-seeking behaviour among selected members of the Indian- immigrant community. It also demonstrates how notions of sexuality are structured by gender and generation, and how these factor impact knowledge about and awareness of HIV/AIDS. This study points to the need for innovative strategies that address social norms and influences among a diverse group of people who maintain close ties with family and friends living abroad. Social network theory can provide guidance for carefully planned peer outreach initiatives which may provide an effective means to reach this underserved community.

\section{Acknowledgements}

Funding for this project was provided by Canadian Institute for Health Research, Grant Number: GGH-84623

\section{References}

1. UNGASS (2010) Country progress report. United Nations.

2. UNAIDS/WHO Working Group on Global HIVIAIDS Surveillance (2008) Epidemiological fact sheet on HIV and AIDS: Core data on epidemiology and response: India.

3. Statistics Canada (2008) Canada's ethno cultural mosaic, 2006 census. Report number 97-562X. ISBN 978-0-662-48324-3

4. Tran K, Kaddatz J, Allard P (2005) South Asians in Canada: Unity through diversity. Canadian Social Trends 78: 20-28.

5. Statistics Canada (2011) Canada Year Book 2011. Catalogue no. 11-402-XP Ottawa, ON: Minister of Industry.

6. Public Health Agency of Canada (2011a) Summary: Estimates of HIV Prevalence and Incidence in Canada, 2011.

7. Public Health Agency of Canada (2011b) At a Glance - HIV and AIDS in Canada: Surveillance Report to December 31st 2011.

8. Ratti R, Bakeman R, Peterson JL (2000) Correlates of high-risk sexua behaviour among Canadian men of South Asian and European origin who have sex with men. AIDS Care12: 193-202.

9. Bhattacharya G (2004) Health Care Seeking for HIVIAIDS among South Asians in the United States. Health Soc Work 29: 106-115.

10. Doshi SR,Gandhi B (2008) Women in India: The context and impact of HIV/ AIDS. Journal of Human Behavior in the Social Environment 17: 413-442.

11. Fourcroy JL (2006) Customs, culture and tradition - what role do they play in a woman's sexuality? J Sex Med 3: 954-959.

12. Fisher W (1997) A theory-based framework for intervention and evaluation in STD/HIV prevention.Canadian Journal of Human Sexuality 6: 105-111.

13. Kohler HP, Behrman JR, Watkins SC (2007) Social networks and HIVIAIDS risk perceptions. Demography 44: 1-33.

14. Latkin CA, Forman V, Knowlton A, Sherman S (2003) Norms, social networks and HIV-related risk behaviors among urban disadvantaged drug users. Soc Sci Med 56: 465-476.

15. Latkin CA, Knowlton AR (2005) Micro-social structural approaches to HIV prevention: a social ecological perspective. AIDS Care: 17: S102-S113.

16. Steward WT, Herek GM, Ramakrishna J, Bharat S, Chandy S, et al. (2008) HIV-related stigma: Adapting a theoretical framework for use in India. Soc Sci Med 67: 1225-1235.

17. Abraham L (2001) Redrawing the Lakshm an Rekha: Gender differences and cultural constructions in youth sexuality in urban India. Journal of South Asian Studies 24: 133-156

18. Chin JJ, Mantell J, Weiss L, Bhagavan M, Lou X (2005) Chinese and South Asian religions institutions and HIV prevention in New York City. AIDS Educ Prev 17: 484-502.

19. Okazaki S (2002) Influences of culture on Asian Americans' sexuality. J Sex Res 39: 34-41.

20. Leininger MM (1978) Transcultural nursing: Concepts, theories, and practices. New York: Wiley.

21. Strauss AL, Corbin J (1998) Basics of Qualitative Research: Grounded Theory Procedures and Techniques. (2ndedn), Thousand Oaks, CA: Sage. 